Preprint

ITP-95-29E

\title{
Unitarized model of hadronic diffractive dissociation
}

\author{
E.S.Martynov, B.V.Struminsky \\ N.N.Bogoliubov Institute for Theoretical Physics, \\ 252143, Metrologicheskaja st. 14b, Kiev-143, Ukraine
}

\begin{abstract}
It is shown that in a supercritical pomeron model the contribution of the tirple-pomeron diagrams violates the unitarity bound for cross-section even with account of the multiple pomeron exchanges between the initial hadrons. Asymptotic behaviour of the single diffractive dissociation cross-section is calculated in the approximation where every pomeron in the $3 P$-diagram is eikonalized as well as an elastic interaction of initial hadrons is taken into account. In this approximation $\sigma^{S D} / \sigma_{\text {tot }} \rightarrow 0$ at $s \rightarrow \infty$.
\end{abstract}


The interest in diffractive dissociation is caused by both comparatively new experimental data obtained at the Tevatron [1] and by observation at HERA of deep inelastic processes with the typical rapidity gaps [2]. Generally adopted interpretation of these similar enough phenomena is based on the following interaction mechanism. The incoming fast proton (or virtual photon in deep inelastic scattering) "emits" a pomeron which interacts with the protontarget producing a shower of hadrons. These hadrons are distributed in the rapidity scale at a large distance (rapidity gap) from the initial proton. The central point of the model, the interaction of pomeron with proton-target, is universal. It means that this subprocess does not depend on where a dissociation is considered: in a pure hadronic process or in a deep inelastic scattering.

In what follows we consider diffractive dissociation in a pure hadronic process $h h \rightarrow h X$. If the effective mass of produced shower is large enough then with certain simplifying assumptions the cross-section of the process may be presented (due to the generalized optical theorem) by the diagram with a triple-pomeron vertex (Fig.1)
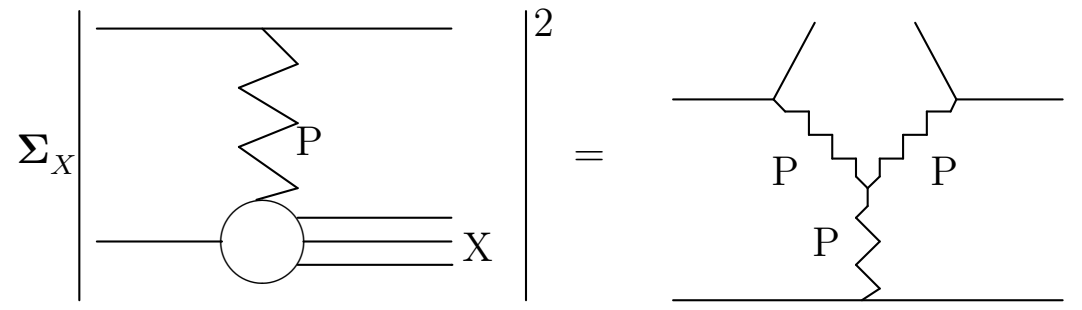

Fig.1 Process of difractive dissociation and 3P-diagramm

The properties of the cross-section of course depend on specific model of pomeron. The most known one at present, the so-called supercritical pomeron has a trajectory with the intercept $\alpha_{P}(0)=1+\Delta$ with $\Delta>0$. In particular the model of A.Donnachie and P.Landshoff with $\Delta=0.08$ based on a pomeron-photon analogy describes quite well the hadronic data [3]. However the contribution of supercritical pomeron to the total cross-section rises with energy as a power $\sigma \propto s^{\Delta}$, violating the Froissart-Martin bound $\sigma_{t o t}<\operatorname{const} \ln ^{2}\left(s / s_{0}\right)$. The strict consistent procedure of unitarization is absent now, but there are some simple phenomenological ways to eliminate the rough contradictions with the unitarity. For example, the eikonal [4], $U$ matrix [5] methods and their generalizations are often used for unitarization of the amplitude. Another approach to the problem was suggested in [6].

It is quite obvious that the three-pomeron diagram needs also for unitarity corrections, which should remove a too fast growing contribution of supercritical pomeron to the diffractive dissociation cross-section (up to the $\ln s$-factors it is proportional to $s^{2 \Delta}$ ). The $3 P$-diagram seemed to be unitarized by the most simple way, taking into account multiple pomeron exchanges between the incoming hadrons (initial state interaction). This approach was suggested in the Ref.[7]. In a more general content a problem of unitarization in diffractive dissociation was discussed a long time ago (see for example [8]). It was claimed in [7] that with the account of the initial state interaction, the integrated cross-section of diffractive dissociation rises logarithmicaly with energy, $\sigma^{S D} \propto \ln s$, in accordance with the experimental data.

We will show that this conclusion of [7] is wrong, and the account of initial state interaction by the eikonal (or by another) way does not allow to eliminate too fast growth of cross-section. 
We have performed the asymptotic evaluations of more wide class of corrections, which indeed allow to eliminate the explicit contradiction with unitarity bounds.

To make our arguments more clear we list a few well known general statements and formulae. Because we are interested only in an asymptotic cross-section behaviour, a contribution of $f$ reggeon is omitted in all expressions.

As in [7] we will work in the impact parameter representation. The normalization of amplitude is

$$
\frac{d \sigma}{d t}=\pi|f(s, t)|^{2}, \quad \sigma_{t o t}=4 \pi \operatorname{Im} f(s, 0) .
$$

An amplitude in $b$-representation is defined by the following transformation

$$
a(s, b)=\frac{1}{2 \pi} \int d \vec{q} e^{-i \vec{q} \vec{b}} f(s, t), \quad t=-q^{2} .
$$

Eikonal summation of the high energy elastic pomeron rescatterings can be realized with the amplitude $a(s, b)$ in the form

$$
a(s, b)=i\left(1-e^{-\Omega(s, b)}\right), \quad \Omega(s, b)=-\frac{i}{2 \pi} \int d \overrightarrow{q e^{-i \vec{q} \vec{b}}} f_{0}(s, t)
$$

where $f_{0}(s, t)$ is an input elastic amplitude. Starting from a simplified model of supercritical pomeron with the trajectory $\alpha_{P}(t)=1+\Delta+\alpha_{P}^{\prime} t$

$$
f_{0}(s, t)=i g(t)\left(\frac{s}{s_{0}}\right)^{\alpha_{P}(t)-1}, \quad g(t)=g e^{-B_{0} t / 4},
$$

one can obtain

$$
\Omega(s, b)=\nu\left(s / s_{0}\right) e^{-b^{2} / R^{2}\left(s / s_{0}\right)},
$$

where

$$
\begin{gathered}
\nu\left(s / s_{0}\right)=\frac{\sigma_{0}}{2 \pi R^{2}\left(s / s_{0}\right)}\left(\frac{s}{s_{0}}\right)^{\Delta}=\frac{4 g^{2}}{R^{2}\left(s / s_{0}\right)}\left(\frac{s}{s_{0}}\right)^{\Delta}, \\
R^{2}\left(s / s_{0}\right)=2 B_{0}+4 \alpha^{\prime} \ln \left(s / s_{0}\right), \quad \sigma_{0}=8 \pi g^{2} .
\end{gathered}
$$

In this model $i f_{0}(s, t)$ and $\Omega(s, b)$ are the real functions, but analiticity and crossing-symmetry are restored by the substitution $s \rightarrow s \exp (-i \pi / 2)$. It is easy to obtain from the above expressions that $\sigma_{t o t} \simeq 2 \pi \Delta R^{2}\left(s / s_{0}\right) \ln \left(s / s_{0}\right)$ at $s / s_{0} \rightarrow \infty$ Thus, in a supercritical pomeron model the eikonal corrections to one-pomeron exchange remove the explicit violation of unitarity condition. The resulting cross-sections satisfies the Froissart-Martin bound.

The expression for an integrated over $t$ cross-section of diffractive dissociation was written in [7] in the form

$$
\begin{gathered}
M^{2} \frac{d \sigma^{S D}}{d M^{2}}=\sigma_{0}^{2} G_{P P P}\left(\frac{s}{M^{2}}\right)^{2 \Delta}\left(\frac{M^{2}}{s_{0}}\right)^{\Delta} \frac{1}{\left[\pi \tilde{R}^{2}\left(s / M^{2}\right)\right]^{2} \pi \tilde{R}^{2}\left(M^{2} / s_{0}\right)} \\
\times \int d \vec{b} d \overrightarrow{b^{\prime}} \exp \left(-2 \nu\left(s / s_{0}\right) e^{-b^{2} / R^{2}\left(s / s_{0}\right)}\right) \exp \left(-2 \frac{\left(\vec{b}-\vec{b}^{\prime}\right)^{2}}{\tilde{R}^{2}\left(s / M^{2}\right)}-\frac{b^{2}}{\tilde{R}^{2}\left(M^{2} / s_{0}\right)}\right),
\end{gathered}
$$

where $\nu, R^{2}$ are defined by exps.(4),(5),

$$
\tilde{R}^{2}(z)=B_{0}+r^{2}+4 \alpha^{\prime} \ln (z)
$$

and $r$ is radius of the triple-pomeron vertex. The eikonal corrections due to pomeron rescatterings in initial state (Fig.2) were accounted by the insertion of factor $\exp (-2 \Omega(s, b))=$ $\exp \left(-2 \nu\left(s / s_{0}\right) e^{-b^{2} / R^{2}\left(s / s_{0}\right)}\right)$ in the integrand of the exp.(6) 


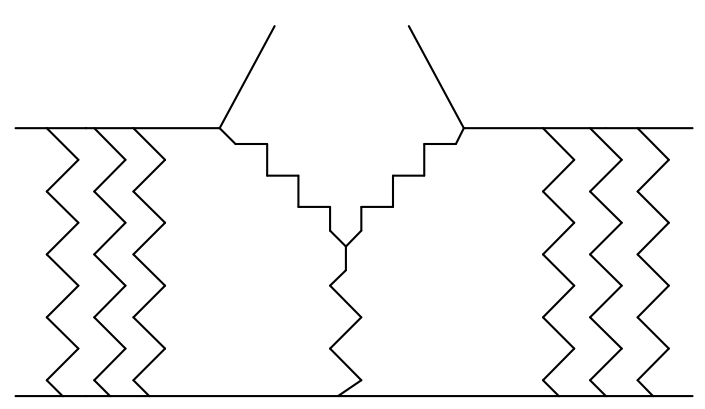

Fig. 2. 3P-diagram with the interaction of hadrons in the initial state

Unfortunately a mistake was appeared in [7] when asymptotic evaluation of the integral was made. After integration over $b$ and $b^{\prime}$ the differential cross-section of diffractive dissociation becomes as following

$$
\frac{M^{2} d \sigma^{S D}}{d M^{2}}=\frac{\sigma_{0}^{2}}{2 \pi \tilde{R}_{1}^{2}\left(s / M^{2}\right)} G_{P P P} \frac{a \gamma[a, 2 \nu(s)]}{[2 \nu(s)]^{a}}\left(\frac{s}{M^{2}}\right)^{2 \Delta}\left(\frac{M^{2}}{s_{0}}\right)^{\Delta},
$$

where

$$
a=\frac{2 R^{2}\left(s / s_{0}\right)}{\tilde{R}^{2}\left(s / M^{2}\right)+2 \tilde{R}^{2}\left(M^{2} / s_{0}\right)},
$$

and $\gamma[a, 2 \nu]$ is the incomplete Euler gamma function.

In the limit under consideration, $s \gg s_{0}, M^{2} / s_{0}, s / M^{2} \gg 1$, the ratio $a$ tends to 2 and $\gamma[a, 2 \nu]$ tends to $\Gamma(2)$. Substituting these limits to the expression (8) authors of [7] have obtained

$$
\frac{M^{2} d \sigma^{S D}}{d M^{2}}=\pi R^{2}\left(s / s_{0}\right) G_{P P P}\left(\frac{M^{2}}{s_{0}}\right)^{-\Delta}
$$

However, this result is wrong. Indeed, one can see using the definitions (4),(5),(7) and (9) that at $s, M^{2}, s / M^{2} \rightarrow \infty$

$$
a=\frac{2 R^{2}\left(s / s_{0}\right)}{\tilde{R}_{1}^{2}\left(s / M^{2}\right)+2 \tilde{R}_{1}^{2}\left(M^{2} / s_{0}\right)}=2\left(1-\frac{\ln \left(M^{2} / s_{0}\right)}{\ln \left(s / s_{0}\right)}+o\left(\frac{1}{\ln \left(s / s_{0}\right)}\right)\right),
$$

Therefore the factor of expression (9) which violates the unitarity is transformed as follows

$$
\left.\left(\frac{s}{M^{2}}\right)^{2 \Delta}\left(\frac{M^{2}}{s_{0}}\right)^{\Delta}[\nu(s)]^{-a} \simeq \exp \left\{2 \Delta \ln \left(\frac{s}{M^{2}}\right)+\Delta \ln \left(\frac{M^{2}}{s_{0}}\right)-2 \Delta \ln \left(s / s_{0}\right)\right]\right\} \sim\left(\frac{M^{2}}{s_{0}}\right)^{\Delta}
$$

conserving too fast growth of cross-section at large $M^{2}$.

In what follows we investigate the diagrams which are important to restore the unitarity. In our opinion it is necessary not only to take into account an interaction of hadrons in the initial state but also to "eikonalize" each pomeron in the diagram of Fig.2. In another words we estimate an asymptotical contribution to $\sigma^{S D}$ of diagrams of Fig.3. 


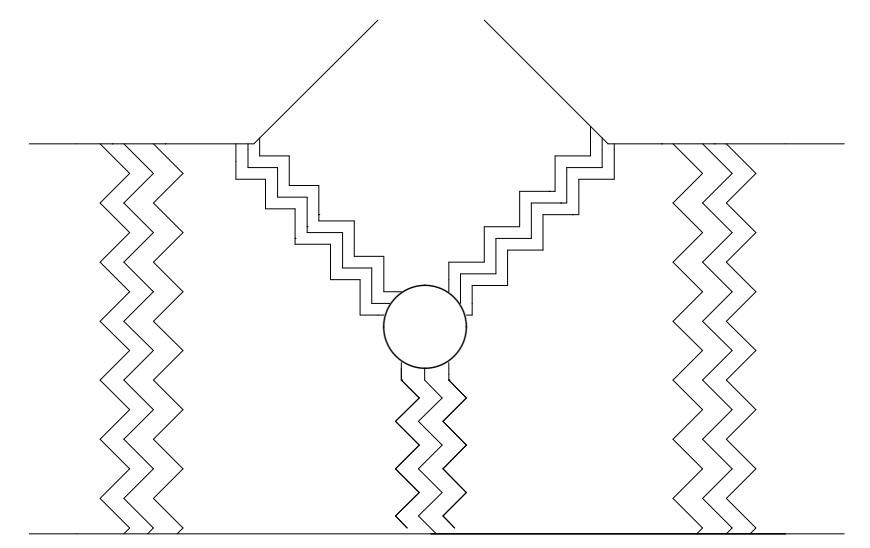

Fig.3. Diagram with the eikonalized pomeron exchanged

Evidently it is impossible to calculate such diagrams in a general form without any simplified assumptions. At the same time there are two important and interesting points. Firstly, does the violation of unitarity bound indeed removed after an eikonalization? Secondly, how fast the eikonalized diffractive cross-section $\sigma^{S D}$ rises at $s \rightarrow \infty$ ?

Our model we define in the form, which corresponds to Fig.3.

$$
M^{2} \frac{d \sigma^{S D}}{d M^{2}}=\sigma_{0}^{-1} \tilde{G} I
$$

where

$$
I=\int d \vec{b} d \overrightarrow{b^{\prime}} \exp (-2 \Omega(s, b))\left\{1-\exp \left[-\tilde{\Omega}\left(s_{0} \frac{s}{M^{2}}, b^{\prime}\right)\right]\right\}^{2}\left\{1-\exp \left[-\tilde{\Omega}\left(M^{2},\left|\vec{b}-\overrightarrow{b^{\prime}}\right|\right)\right]\right\}
$$

and $\tilde{G}$ includes all of the relevant couplings and constants. The value $\tilde{\Omega}(z, b)$ differs of the input one-pomeron $\Omega(z, b)$, (exp. (4)). The difference is that one of vertices $g$ (in $s, t$-representation) is changed for the part of triple-pomeron vertex.

$$
V_{P P P}\left(t_{0} ; t_{1}, t_{2}\right)=v \exp \left(r_{0}^{2} t_{0}+r_{1,2}^{2}\left(t_{1}+t_{2}\right)\right)
$$

specifically

$$
g\left(t_{i}\right) \rightarrow v^{1 / 3} \exp \left(r_{i}^{2} t_{i}\right)
$$

We note that it is not enough to eikonalize only pomerons in $3 P$-vertex in order to restore unitarity. If we do that, the factor $\exp (-2 \Omega(s, b))$ is absent in the integrand of (10). Integral is calculated easily

$$
\begin{gathered}
M^{2} \frac{d \sigma^{S D}}{d M^{2}} \propto R_{1}^{2} R_{2}^{2} \ln \left(s / M^{2}\right) \ln \left(M^{2} / s_{0}\right), \\
\sigma^{S D} \propto \ln ^{5}\left(s / s_{0}\right) .
\end{gathered}
$$

The result evidently contradicts to the Froissart-Martin bound.

Let's now calculate the asymptotic behaviour of integral $I$ at the limit $s \rightarrow \infty$. To do that let's rewrite it in the following form

$$
\begin{aligned}
I=4 \pi \int_{0}^{\infty} d b \int_{0}^{\infty} d b^{\prime} \int_{0}^{\pi} d \phi b b^{\prime}\left[1-e^{-\nu_{1} e^{-b^{\prime 2} / R_{1}^{2}}}\right]\left[1-e^{-\nu_{2} e^{-b^{2} / R_{2}^{2}}}\right]^{2} \\
\times \exp \left\{-2 \nu_{0} \exp \left[-\left(b^{2}+b^{\prime 2}+2 b b^{\prime} \cos \phi\right) / R_{0}^{2}\right]\right\} .
\end{aligned}
$$


To avoid the extra cumbersations in the following formulae we use the notations:

$$
\begin{gathered}
\nu_{0} \equiv \nu\left(s / s_{0}\right), \quad \nu_{1}=\frac{4 g v}{R_{1}^{2}}\left(\frac{M^{2}}{s_{0}}\right)^{\Delta}, \quad \nu_{2}=\frac{4 g v}{R_{2}^{2}}\left(\frac{s}{M^{2}}\right)^{\Delta}, \\
R_{0}^{2} \equiv R^{2}\left(s / s_{0}\right), \quad R_{1}^{2} \equiv \tilde{R}_{1}^{2}\left(M^{2} / s_{0}\right), \quad R_{2}^{2} \equiv \tilde{R}_{2}^{2}\left(s / M^{2}\right),
\end{gathered}
$$

where $R^{2}\left(s / s_{0}\right)$ is defined by exp.(5), and

$$
\tilde{R}_{1}^{2}\left(M^{2} / s_{0}\right)=B_{0}+r_{0}^{2}+4 \alpha^{\prime} \ln \left(M^{2} / s_{0}\right), \quad \tilde{R}_{2}^{2}\left(s / M^{2}\right)=B_{0}+r_{1,2}^{2}+4 \alpha^{\prime} \ln \left(s / M^{2}\right) .
$$

Making the substitution of the integration variables

$$
\nu_{1} \exp \left(-b^{2} / R_{1}^{2}\right)=z_{1}, \quad \nu_{2} \exp \left(-b^{2} / R_{2}^{2}\right)=z_{2}
$$

we get

$$
\begin{aligned}
I= & 2 \pi R_{1}^{2} R_{2}^{2} \int_{0}^{\nu_{1}} \frac{d z_{1}}{z_{1}} \int_{0}^{\nu_{2}} \frac{d z_{2}}{z_{2}}\left(1-e^{-z_{1}}\right)^{2}\left(1-e^{-z_{2}}\right) \\
& \times \int_{0}^{\pi} d \phi \exp \left\{-2 \nu_{0} \exp \left[-\left(\rho_{1} \ln \left(\frac{\nu_{1}}{z_{1}}\right)+2 \sqrt{\rho_{1} \ln \left(\frac{\nu_{1}}{z_{1}}\right) \rho_{2} \ln \left(\frac{\nu_{2}}{z_{2}}\right)} \cos \phi+\rho_{2} \ln \left(\frac{\nu_{2}}{z_{2}}\right)\right)\right]\right\},
\end{aligned}
$$

where

$$
\rho_{1}=\tilde{R}_{1}^{2} / R_{0}^{2}, \quad \rho_{2}=\tilde{R}_{2}^{2} / R_{0}^{2}
$$

It is convenient to divide both integrals over $z_{1}$ and $z_{2}$ into two parts: one is from 0 up to 1 and second is from 1 up to $\nu_{i}$.

$$
I=I_{0,0}^{1,1}+I_{1,0}^{\nu_{1}, 1}+I_{0,1}^{1, \nu_{2}}+I_{1,1}^{\nu_{1}, \nu_{2}}
$$

Consider for example first of them where the integrations over z's go from 0 up to 1 (remaining ones are similarly estimated). The small $z_{1}$ and $z_{2}$ contribute to it. Therefore replacing $1-$ $\exp \left(-z_{i}\right)$ by $z_{i}$ and using the new variables

$$
x_{i}=-\frac{\ln z_{i}}{\ln \nu_{i}}, \quad i=1,2
$$

we write $I_{0,0}^{1,1}$ in the form

$$
\begin{aligned}
I_{0,0}^{1,1}= & 2 \pi R_{1}^{2} R_{2}^{2} \ln \nu_{1} \ln \nu_{2} \int_{0}^{\pi} d \phi \int_{0}^{\infty} d x_{1} \exp \left(-2 x_{1} \ln \nu_{1}\right) \int_{0}^{\infty} d x_{2} \exp \left(-x_{2} \ln \nu_{2}\right) \\
& \times \exp \left\{-2 \nu_{0} \exp \left[-\left(\beta_{1} \sqrt{1+x_{1}}+\beta_{2} \sqrt{1+x_{2}}\right)^{2}+4 \beta_{1} \beta_{2} \sqrt{\left(1+x_{1}\right)\left(1+x_{2}\right)} \sin ^{2} \frac{\phi}{2}\right]\right\}
\end{aligned}
$$

where

$$
\beta_{1}=\sqrt{\rho_{1} \ln \nu_{1}}, \quad \beta_{2}=\sqrt{\rho_{2} \ln \nu_{2}} .
$$

Before the further calculations let's pay attention to the behaviour of the $\rho, \beta, \nu$ at $s \rightarrow \infty$. It is easy to see that for an arbitrary but fixed $\rho_{i}$

$$
\ln \nu_{i}=\rho_{i} \ln \nu_{0} \cdot\left\{1-\frac{\ln \left(R_{0}^{2} / \sigma_{0}\right)}{\ln \nu_{0}}+o\left(\frac{1}{\ln \left(s / s_{0}\right)}\right)\right\}
$$




$$
\begin{gathered}
\beta_{i}=\rho_{i} \sqrt{\ln \nu_{0}} \cdot\left\{1-\frac{1}{2} \frac{\ln \left(R_{0}^{2} / \sigma_{0}\right)}{\ln \nu_{0}}+o\left(\frac{1}{\ln \left(s / s_{0}\right)}\right)\right\} \\
\rho_{1}+\rho_{2}=1+\frac{r_{0}^{2}+r_{1,2}^{2}}{R_{0}^{2}}, \quad\left(\beta_{1}+\beta_{2}\right)^{2}=\ln \nu_{0}-\ln \left(\frac{R_{1}^{2} R_{2}^{2}}{\sigma_{0} R_{0}^{2}}\right)+o(1) .
\end{gathered}
$$

Making use of above properties of $\rho, \beta, \nu$, one can argue that the main contribution in the integrals over $x_{i}, \phi$ is determined by small $x_{1}, x_{2}, \phi$. Keeping the linear in $x_{1}, x_{2}, \phi^{2}$ terms in the internal exponential and substituting

$$
u_{1}=\exp \left(-\beta_{1}\left(\beta_{1}+\beta_{2}\right) x_{1}\right), \quad u_{2}=\exp \left(-\beta_{2}\left(\beta_{1}+\beta_{2}\right) x_{2}\right),
$$

we obtain

$$
\begin{aligned}
I_{0,0}^{1,1}= & 2 \pi \frac{R_{1}^{2} R_{2}^{2} \ln \nu_{1} \ln \nu_{2}}{\beta_{1} \beta_{2}\left(\beta_{1}+\beta_{2}\right)^{2}} \int_{0}^{\pi} d \phi \int_{0}^{1} \frac{d u_{1}}{u_{1}} \int_{0}^{1} \frac{d u_{2}}{u_{2}} u_{1}^{a_{1}} u_{2}^{a_{2}} \exp \left\{-2 \frac{R_{1}^{2} R_{2}^{2}}{\sigma_{0} R_{0}^{2}} \exp \left(\beta_{1} \beta_{2} \phi^{2}\right) u_{1} u_{2}\right\} \simeq \\
& 2 \pi R_{1}^{2} R_{2}^{2} \frac{\sqrt{\pi} \ln \nu_{1} \ln \nu_{2}}{\left(\beta_{1} \beta_{2}\right)^{3 / 2}\left(\beta_{1}+\beta_{2}\right)^{2}\left(a_{1}-a_{2}\right)}\left\{\frac{\Gamma\left(a_{2}\right)}{\sqrt{a_{2}}}\left(2 \frac{R_{1}^{2} R_{2}^{2}}{\sigma_{0} R_{0}^{2}}\right)^{-a_{2}}-\frac{\Gamma\left(a_{1}\right)}{\sqrt{a_{1}}}\left(2 \frac{R_{1}^{2} R_{2}^{2}}{\sigma_{0} R_{0}^{2}}\right)^{-a_{1}}\right\},
\end{aligned}
$$

where

$$
a_{1}=\frac{\beta_{1} / \rho_{1}}{\beta_{1}+\beta_{2}}, \quad a_{2}=\frac{2 \beta_{2} / \rho_{2}}{\beta_{1}+\beta_{2}} .
$$

Since $a_{1}=1+o\left(1 / \ln \left(s / s_{0}\right)\right)$ and $a_{2}=2+o\left(1 / \ln \left(s / s_{0}\right)\right)$, as it follows from (12),(13), we finally obtain for integral $I_{0,0}^{1,1}$

$$
I_{0,0}^{1,1}=\pi \sqrt{\pi} \sigma_{0} R_{0}^{2} \frac{\ln \nu_{1} \ln \nu_{2}}{\left(\beta_{1} \beta_{2}\right)^{3 / 2}\left(\beta_{1}+\beta_{2}\right)^{2}}\left(1+o\left(\frac{1}{\ln \left(s / s_{0}\right)}\right)\right) .
$$

The similar calculations for another terms of integral $I$ give rise to the following results

$$
I_{0,1}^{1, \nu_{2}} \simeq I_{0,0}^{1,1}, \quad I_{1,0}^{\nu_{1}, 1} \simeq \frac{1}{2 \sqrt{2}} \cdot \frac{\sigma_{0} R_{0}^{2}}{R_{1}^{2} R_{2}^{2}} I_{0,0}^{1,1}, \quad I_{1,1}^{\nu_{1}, \nu_{2}}<\text { const } \exp \left(-R_{0}^{2} / \sigma_{0}\right) .
$$

Thus at $s \rightarrow \infty$

$$
M^{2} \frac{d \sigma^{S D}}{d M^{2}} \simeq \pi \sqrt{\pi} \tilde{G}\left(R_{0}^{2}\right)^{2} \sqrt{\frac{\Delta \ln \left(s / s_{0}\right)}{R_{1}^{2} R_{2}^{2}}} \simeq \text { const } \frac{\left[\ln \left(s / s_{0}\right)\right]^{3 / 2}}{\sqrt{\ln \left(s / M^{2}\right) \ln \left(M^{2} / s_{0}\right)}}
$$

Integration of the exp.(14) over $M^{2}$ in the domain where $\rho_{1,2} \not \rightarrow 0$, gives

$$
\sigma^{S D} \propto \ln ^{3 / 2}\left(s / s_{0}\right), \quad \sigma^{S D} / \sigma_{t o t} \rightarrow 0 \quad \text { at } \quad s \rightarrow \infty .
$$

Thus we have proposed and investigated the simplified eikonal model for a process of hadronic single diffractive dissociation. We have shown that eikonalization of each pomeron in the 3P-diagram and account of the elastic interaction of hadrons in the initial state allow to restore the unitarity which is violated by an input supercritical pomeron. Our result is only asymptotical one. The numerical calculations and account of the nonasymptotical contributions are needed to compare the considered model with experiment.

We thank very much A.I.Bugrij and L.L.Jenkovszky for the useful discussions on the considered problem.

The research was made possible in part by Grant K4N100 from the Joint Fund of the Government of Ukraine and International Science Foundation. 


\section{References}

1. N.Amos et al., Phys. Lett. B301, 313 (1993);

CDF Collaboration: F.Abe et al., Phys. Rev. D50, 5535 (1994).

2. ZEUS Collaboration: M.Derric et al., Phys. Lett. B338, 483 (1994);

H1 Collaboration: T.Ahmed et al., Phys. Lett. B348, 681 (1995).

3. A.Donnachie and P.Lahdshoff, Nucl. Phys. 231, 189 (1984); Phys. Lett. B296, 227, 1992.

4. T.T.Chou and C.N.Yang, Phys. Rev. 170, 1591 (1968).

5. O.A.Khrustaliov, V.E.Savrin and N.E.Tyurin, Elementary Particles and Atomic Nuclear 7, 21 (1976).

6. E.M.Levin, Phys. Rev. D49, 4469 (1994).

7. E.Gotsman, E.M.Levin and U.Maor, Phys.Rev. D49, 4321 (1994).

8. A.Capella, J.Kaplan and J.Tran Thanh Van, Nucl. Phys. B105, 333 (1976). 